\title{
Fatores assistenciais e gestacionais associados à anemia em nutrizes atendidas em um banco de leite humano
}

\author{
Healthcare and gestational factors associated with anemia \\ in nursing mothers attended in a human milk bank
}

\author{
Larissa Bueno Ferreira ${ }^{1}$ \\ Luisa Freitas de Melo ${ }^{1}$ \\ Maria Eduarda Fernandes de Melo ${ }^{1}$ \\ Taciana Maia de Sousa ${ }^{1}$ \\ Cristianny Miranda ${ }^{1}$ \\ Simone Cardoso Lisboa Pereira ${ }^{1}$ \\ Karine Antunes Marques Notaro ${ }^{1}$ \\ Luana Caroline dos Santos ${ }^{1}$
}

${ }^{1}$ Departamento de Nutrição, Universidade Federal de Minas Gerais. Av. Alfredo Balena 190, Santa Efigênia. 30130-100 Belo Horizonte MG Brasil. labuenoferreira@gmail.com
Abstract Anemia is a public health problem that affects about $30 \%$ of Brazilian women, which may cause deleterious effects on the health and quality of life of mother and child. This article seeks to evaluate the healthcare and gestational factors associated with anemia among mothers receiving care at a human milk bank (HMB) of reference. It is a cross-sectional study with secondary data about sociodemographic and pregnancy information on 12283 nursing mothers from 2009 to 2012. The Kolmogorov-Smirnov and chi-square or Fisher's exact tests were applied. The prevalence ratios $(P R)$ and their 95\% confidence intervals were estimated by Poisson regression with robust variance. Anemia was identified in $29.2 \%$ of the sample and the prevalence was higher among mothers who did not have prenatal appointments $(P R=3.84 ; 95 \%$ CI 3.26 to 4.54); those who made 3 or less antenatal visits ( $P R=1.92 ; 95 \%$ CI 1.21 to 3.06) and those who have had multiple pregnancies $(O R=2.29,95 \%$ CI 1.25 to 4.19$)$. The results showed a higher prevalence of anemia among women who did not have the appropriate prenatal care and who have had multiple pregnancies, highlighting the importance of healthcare as a factor in the prevention of anemia and other complications during pregnancy.

Key words Pregnancy, Anemia, Milk bank, Healthcare, Prenatal care
Resumo A anemia é um problema de saúde pública que acomete cerca de $30 \%$ das gestantes brasileiras, podendo acarretar efeitos deletérios à saúde e qualidade de vida do binômio mãefilho. O objetivo deste artigo é avaliar os fatores assistenciais e gestacionais associados à anemia entre nutrizes atendidas em um banco de leite humano (BLH) de referência. Estudo transversal com dados secundários referentes a informações sociodemográficas e gestacionais de 12283 nutrizes no período de 2009 a 2012. Aplicaram-se os testes Kolmogorov-Smirnov e Qui-quadrado ou exato de Fisher. Estimaram-se as Razões de Prevalência $(R P)$ e respectivos Intervalos de Confiança-IC95\%, por meio da Regressão de Poisson com variância robusta. Identificou-se a prevalência de anemia em 29,2\% da amostra, sendo maior entre as mães que não realizaram o pré-natal ( $R P$ $=3,84$; IC95\% 3,26-4,54); as que realizaram até 3 consultas pré-natais $(R P=1,92$; IC95\% 1,21$3,06)$ e aquelas que tiveram gestação múltipla ( $R P$ $=2,29$; IC95\% 1,25-4,19). Os resultados apontaram maior prevalência de anemia entre as mulheres que não realizaram o pré-natal apropriado $e$ as que tiveram gestações múltiplas, destacando-se a importância da assistência à saúde como fator de prevenção à anemia e demais intercorrências gestacionais.

Palavras-chave Gestação, Anemia, Bancos de Leite, Atenção à saúde, Cuidado pré-natal 


\section{Introdução}

A anemia pode ser definida como um estado em que a concentração de hemoglobina do sangue é anormalmente baixa em consequência da carência de um ou mais nutrientes essenciais ${ }^{1}$. Todavia, sabe-se que, entre as diversas causas de anemia descritas na literatura, a deficiência de ferro, que resulta na chamada anemia ferropriva, é a mais comum. Esse agravo ocorre em razão de um balanço negativo entre a quantidade de ferro biodisponível e a necessidade desse nutriente em um longo período de tempo, acometendo principalmente crianças, gestantes e nutrizes ${ }^{1,2}$.

Segundo um estudo publicado em 2015, pela Organização Mundial de Saúde ${ }^{1}$, estima-se que mais da metade das gestantes sejam anêmicas nos países em desenvolvimento, enquanto nos países desenvolvidos essa situação acomete cerca de um quarto das grávidas. Já no Brasil, o Ministério da Saúde (MS) indica prevalência de $30 \%{ }^{1}$. Em virtude deste cenário, a redução da anemia está inserida entre as metas globais de nutrição para 2025, com perspectiva de declínio de 50\% entre as mulheres em idade reprodutiva ${ }^{1-3}$.

Os altos valores de prevalência de anemia durante a gestação estão relacionados às adaptações fisiológicas que incluem aumento de aproximadamente $50 \%$ do nível plasmático para suprir a hipertrofia vascular do útero em expansão durante a gravidez ${ }^{4}$. Apesar da redução na depleção do ferro em virtude da amenorreia gestacional e concomitante aumento na absorção intestinal desse mineral, ainda há grande demanda de ferro na gestação, sendo esta de 0,8 até $7,5 \mathrm{mg} /$ dia entre o primeiro até o final do terceiro trimestre ${ }^{4,5}$.

As consequências da anemia na gestação, assim como sua causalidade multifatorial, são complexas tendo em vista possíveis efeitos deletérios à saúde e qualidade de vida do binômio mãe-filho ${ }^{6}$. A presença desta condição favorece o incremento da taxa de mortalidade materna e perinatal; maior risco de prematuridade, baixo peso ao nascer e reservas de ferro abaixo do normal entre os recém-nascidos ${ }^{5,6}$.

A nutrição adequada, com oferta apropriada de ferro, somada à suplementação deste mineral é recomendada em todo o mundo durante o período gestacional, a fim de ajustar o seu status e prevenir a sua deficiência ${ }^{1}$. Cumpre ainda salientar que a deficiência de outros nutrientes (vitamina A e zinco, por exemplo) pode favorecer o quadro de anemia ${ }^{7}$, assim como situações clínicas como inflamação crônica, distúrbios hereditários e doenças infecciosas (malária e parasitas intesti- nais), frequentemente mediadas pelas condições socioeconômicas da gestante e acesso incipiente aos serviços de saúde durante o acompanhamento gestacional ${ }^{8}$.

O pré-natal se configura como um momento importante de promoção à saúde da gestante e do feto e tem como objetivo a prevenção dos determinantes da morbimortalidade materno-infantil, diminuindo os agravos comuns ao período da gestação, incluindo a anemia ${ }^{8,9}$. Assim, demanda-se, neste período, uma abordagem ampla que identifique todos os possíveis fatores causais da anemia, aspectos promotores da sua continuidade e que possam comprometer o seu controle efetivo $^{4,10}$.

O MS considera como ideal a realização de, no mínimo, seis consultas durante a gestação ${ }^{11}$, no entanto, observa-se no Brasil, uma baixa qualidade do pré-natal associada à menor renda familiar, idade materna inferior a 18 anos, menor escolaridade materna e ausência de companheiro ${ }^{12}$.

Diante do exposto, este artigo objetivou avaliar os fatores assistenciais e gestacionais associados à anemia gestacional em nutrizes atendidas em um banco de leite humano (BLH) de referência.

\section{Metodologia}

Trata-se de um estudo transversal com dados secundários, obtidos de formulários estruturados do próprio serviço, com os dados coletados no período de 2009 a 2012 em um BLH de uma maternidade referência de Belo Horizonte, Minas Gerais.

Para a presente investigação foram utilizadas informações sociodemográficas (idade, endereço, profissão e estado civil), assistenciais (local do pré-natal, número de consultas, orientações sobre o aleitamento materno - AM no pré-natal) e gestacionais maternas (hemoglobina no período da gestação, tipo de parto, idade gestacional, peso ao nascer, morbidade referida, transfusão de sangue, gestação múltipla e experiência prévia em $\mathrm{AM})$ em nutrizes atendidas no BLH.

Para fins de análise, as consultas pré-natais realizadas pelas mães foram classificadas em três categorias: (1) não realizou, e até três consultas, (2) de quatro a seis consultas e (3) seis ou mais consultas pré-natais, essa categorização foi realizada para melhor distribuição das participantes entre os grupos, uma vez que houve baixa adesão ao pré-natal na amostra estudada. No entanto, ressalta-se que o número ideal de consultas, segundo o MS é de no mínimo seis ${ }^{11}$. 
Já a idade gestacional foi categorizada em pré-termo (menos de 37 semanas completas de gestação) e a termo (de 37 semanas a menos de 42 semanas completas de gestação) ${ }^{13}$. Em relação à idade da nutriz, esta foi categorizada em quatro faixas: menores de 20 anos (adolescentes); adultas jovens, com idade entre 20 a 29 anos; adultas, com idade entre 30 a 39 anos e adultas, com 40 anos ou mais ${ }^{14,15}$.

Quanto ao estado civil, foram consideradas mulheres casadas aquelas com qualquer forma de relação estável com o parceiro e, no que se refere às concentrações de hemoglobina, as mesmas foram consideradas normais quando incluídas no intervalo de 12 a $16 \mathrm{~g} / \mathrm{dL}^{16}$. Ressalta-se que essas informações foram coletas a partir dos exames de sangue apresentados no momento do atendimento no BLH.

Os dados obtidos foram organizados no programa Microsoft Office Excel versão 2007 e analisados com auxílio dos softwares Statistical Package for the Social Sciences ${ }^{\circledR}$ (SPSS), versão 19.0, e Stata ${ }^{\circledR}$ versão 11.2. Após análise de consistência, as variáveis quantitativas foram testadas quanto à adesão à distribuição normal por meio do teste Kolmogorov-Smirnov e foram realizadas estimativas das medidas de frequências (absolutas e relativas), tendência central (médias e medianas) e de dispersão (desvio-padrão, valores mínimo e máximo). Ademais, foram aplicados os testes Qui-quadrado ou exato de Fisher para comparação de proporções.

Por fim, foram estimadas as Razões de Prevalência (RP) da presença de anemia, com os respectivos Intervalos de Confiança-IC95\% por meio da Regressão de Poisson com variância robusta. Foram incluídas no modelo multivariado as variáveis com valor $\mathrm{p}<0,20$ na análise univariada. O método backward foi utilizado para eliminação das variáveis e o teste Hosmer e Lemeshow empregado para ajuste do modelo final. Para todas as análises foi adotado o nível de significância de 5\% $(\mathrm{p}<0,05)$.

Quanto aos aspectos éticos, ressalta-se que esse estudo faz parte do projeto "Banco de leite humano de referência em Minas Gerais: caracterização, intervenção e avaliação", aprovado pelos Comitês de Ética envolvidos.

\section{Resultados}

No total foram avaliadas 12283 nutrizes, sendo que $83,1 \%$ apresentavam entre 20 e 39 anos e $60,8 \%$ eram casadas. Além disso, $74,8 \%$ relata- ram trabalho remunerado e 71,6\% residiam na capital mineira. Em relação às variáveis assistenciais, $98,7 \%$ afirmaram ter realizado pré-natal, das quais $53,1 \%$ fizeram o acompanhamento no serviço público de saúde e $86,6 \%$ relataram ter realizado mais de seis consultas. Demais dados de caracterização estão apresentados na Tabela 1.

A prevalência de anemia gestacional foi identificada em $29,2 \%$ da amostra e foi associada, na análise univariada, com características sociodemográficas da mulher, como profissão $(\mathrm{p}=$ $0,008)$, estado civil $(p=0,023)$, número de consultas pré-natal $(\mathrm{p}=0,005)$ e gestação múltipla $(\mathrm{p}=0,043)$ (Tabela 2). Além disso, as variáveis "local de realização do pré-natal" $(\mathrm{p}=0,186)$ e "idade" ( $p=0,205)$ também foram recrutadas para análise multivariada, por apresentarem valor p inferior ou aproximado a 0,20 .

$\mathrm{Na}$ análise multivariada, comparando-se com mulheres que realizaram ao menos seis consultas pré-natais, maiores prevalências de anemia foram verificadas entre aquelas que não realizaram ou compareceram em até três consultas pré-natais $(60,0 \%$ vs. $29,1 \%$; $p=0,153)$, simultaneamente. Mulheres com gestação múltipla tiveram maior prevalência de anemia em relação às gestantes não gemelares $(62,5 \%$ vs $27,6 \% ; \mathrm{p}=$ $0,043)$, assim como entre aquelas que realizaram até três consultas pré-natais, de quatro a seis consultas e seis ou mais ( $53,6 \%$ vs $35,0 \%$ vs $28,0 \%$; p $=0,005)$, respectivamente (Tabela 3 ).

\section{Discussão}

O presente estudo encontrou uma prevalência moderada de anemia gestacional $(29,2 \%)$, segundo parâmetros da Organização Mundial de Saúde $(\mathrm{OMS})^{17}$, sendo esta condição associada a fatores assistenciais (pré-natal) e gestacionais (gestação múltipla) ${ }^{17}$. Adicionalmente, a prevalência identificada foi próxima das estimativas nacionais ( $30 \%$ a $40 \%)$ e destaca a anemia como um importante problema de saúde pública com potenciais repercussões negativas para a saúde da gestante e do bebề

Diante desse cenário, o governo brasileiro implantou em 2005 o Programa Nacional de Suplementação de Ferro, revisto em 2013, que preconiza que todas as gestantes e mulheres até o terceiro mês pós-parto devem receber a suplementação de forma profilática, sendo a dosagem recomendada de $40 \mathrm{mg}$ de ferro elementar por dia $^{18-20}$. Além disso, o governo adotou também a obrigatoriedade da fortificação das farinhas de 
Tabela 1. Características sociodemográficas, assistenciais e gestacionais da amostra estudada referente à anemia. Banco de leite humano. Belo Horizonte/MG, Brasil, 2009 - 2012.

\begin{tabular}{|c|c|c|c|}
\hline Variáveis & Categorias & $\mathbf{N}$ & Percentual (\%) \\
\hline \multicolumn{4}{|l|}{ Sociodemográficas } \\
\hline \multirow[t]{4}{*}{ Idade } & $<20$ anos & 1522 & 13,0 \\
\hline & 20 a 29 anos & 4780 & 41,0 \\
\hline & 30 a 39 anos & 4916 & 42,1 \\
\hline & 40 anos ou mais & 450 & 3,9 \\
\hline \multirow[t]{3}{*}{ Local de residência } & Belo Horizonte & 7161 & 71,6 \\
\hline & Região metropolitana & 2374 & 23,8 \\
\hline & Demais cidades & 460 & 4,6 \\
\hline \multirow[t]{3}{*}{ Profissão } & Do lar & 1927 & 19,8 \\
\hline & Estudante & 524 & 5,4 \\
\hline & Remunerada & 7257 & 74,8 \\
\hline \multirow[t]{3}{*}{ Estado civil } & Solteira & 4123 & 38,0 \\
\hline & Casada & 6595 & 60,8 \\
\hline & Outros & 131 & 1,2 \\
\hline \multicolumn{4}{|l|}{ Assistenciais } \\
\hline \multirow[t]{3}{*}{ Pré-natal } & Sim, público & 6181 & 52,4 \\
\hline & Sim, privado & 5468 & 46,3 \\
\hline & Não & 150 & 1,3 \\
\hline \multirow{3}{*}{ No consultas pré-natais } & Até 3 & 285 & 2,6 \\
\hline & De 4 a 6 & 1168 & 10,8 \\
\hline & Acima de 6 & 9349 & 86,6 \\
\hline \multirow[t]{3}{*}{ Orientações sobre AM no pré-natal } & Sim, individual & 2410 & 23,9 \\
\hline & Sim, em grupo & 1496 & 14,9 \\
\hline & Não & 6168 & 61,2 \\
\hline \multicolumn{4}{|l|}{ Gestacionais } \\
\hline \multirow[t]{2}{*}{ Experiência prévia em AM } & Sim & 2851 & 38,9 \\
\hline & Não & 4478 & 61,1 \\
\hline \multirow[t]{2}{*}{ Morbidade referida } & Sim & 1967 & 20,6 \\
\hline & Não & 7573 & 79,4 \\
\hline \multirow[t]{2}{*}{ Idade gestacional } & RN pré-termo & 2384 & 27,3 \\
\hline & $\mathrm{RN}$ a termo & 6339 & 72,7 \\
\hline \multirow[t]{2}{*}{ Hemoglobina } & Normal & 768 & 70,8 \\
\hline & Abaixo do VR & 317 & 29,2 \\
\hline \multirow[t]{2}{*}{ Transfusão } & Sim & 150 & 2,3 \\
\hline & Não & 6421 & 97,7 \\
\hline \multirow[t]{3}{*}{ Tipo de parto } & Normal & 4905 & 41,6 \\
\hline & Cesárea & 6574 & 55,7 \\
\hline & Fórceps & 319 & 2,7 \\
\hline \multirow[t]{2}{*}{ Peso ao nascer } & Baixo peso & 1532 & 16,5 \\
\hline & Peso adequado & 7755 & 83,5 \\
\hline \multirow[t]{2}{*}{ Gestação múltipla } & Sim & 85 & 0,9 \\
\hline & Não & 9202 & 99,1 \\
\hline
\end{tabular}

Nota: AM: Aleitamento materno; RN: Recém-nascido; VR: valor de referência.

trigo e milho como outra medida para minimizar a anemia (RDC no 344, de 2002) ${ }^{18}$.

Estima-se que essa estratégia de fortificação dos grãos possa contribuir para 67\% de declínio da anemia ferropriva e favoreça a queda de 19\% na incidência de baixo peso ao nascer ${ }^{19}$. De acordo com a Organização Mundial da Saúde, a suplementação diária de ferro no período gestacional reduziu o risco de anemia materna em $70 \%$ e deficiência de ferro em $57 \%{ }^{17,21}$. No entanto, a 
Tabela 2. Fatores associados à classificação de anemia da amostra estudada. Banco de leite humano. Belo Horizonte/MG, Brasil, 2009 - 2012.

\begin{tabular}{|c|c|c|c|c|}
\hline \multirow{2}{*}{ Variáveis } & \multicolumn{4}{|c|}{ Hemoglobina } \\
\hline & Não anêmicas (\%) & Anêmicas (\%) & $\mathrm{P}^{\mathrm{a}}$ & RP \\
\hline \multicolumn{5}{|c|}{ 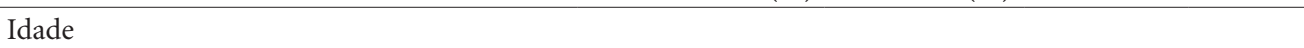 } \\
\hline$<20$ anos & 63,9 & 36,1 & 0,205 & 1,08 \\
\hline 20 a 29 anos & 71,9 & 28,1 & & 0,84 \\
\hline 30 a 39 anos & 72,0 & 28,0 & & 0,84 \\
\hline 40 anos ou mais & 66,7 & 33,3 & & 1,00 \\
\hline \multicolumn{5}{|l|}{ Residência } \\
\hline Belo Horizonte & 71,9 & 28,1 & 0,634 & 0,99 \\
\hline Região metropolitana & 68,5 & 31,5 & & 1,11 \\
\hline Demais cidades & 71,6 & 28,4 & & 1,00 \\
\hline \multicolumn{5}{|l|}{ Profissão } \\
\hline Do lar & 63,3 & 36,7 & 0,008 & 1,45 \\
\hline Estudante & 70,0 & 30,0 & & 1,18 \\
\hline Remunerada & 74,7 & 25,3 & & 1,00 \\
\hline \multicolumn{5}{|l|}{ Estado civil } \\
\hline Solteira & 66,7 & 33,3 & 0,019 & 0,78 \\
\hline Casada & 74,6 & 25,4 & & 0,59 \\
\hline Outros & 57,1 & 42,9 & & 1,00 \\
\hline \multicolumn{5}{|l|}{ Pré-natal } \\
\hline Sim, público & 69,7 & 30,3 & 0,186 & 1,00 \\
\hline Sim, privado & 72,7 & 27,3 & & 0,90 \\
\hline Não & 40,0 & 60,0 & & 1,98 \\
\hline \multicolumn{5}{|l|}{ No consultas pré-natais } \\
\hline Até 3 & 46,4 & 53,6 & 0,005 & 1,92 \\
\hline De 4 a 6 & 65,0 & 35,0 & & 1,25 \\
\hline Acima de 6 & 72,0 & 28,0 & & 1,00 \\
\hline \multicolumn{5}{|c|}{ Orientações sobre AM no pré-natal } \\
\hline Sim, individual & 72,9 & 27,1 & 0,587 & 0,89 \\
\hline Sim, em grupo & 68,3 & 31,7 & & 1,05 \\
\hline Não & 69,7 & 30,3 & & 1,00 \\
\hline \multicolumn{5}{|l|}{ Experiência prévia em AM } \\
\hline Sim & 66,3 & 33,7 & 0,249 & 1,14 \\
\hline Não & 70,6 & 29,4 & & 1,00 \\
\hline \multicolumn{5}{|l|}{ Morbidade referida } \\
\hline Sim & 71,6 & 28,4 & 0,788 & 0,96 \\
\hline Não & 70,3 & 29,7 & & 1,00 \\
\hline \multicolumn{5}{|l|}{ Transfusão } \\
\hline Sim & 76,9 & 23,1 & 1,000 & 0,81 \\
\hline Não & 71,6 & 28,4 & & 1,00 \\
\hline \multicolumn{5}{|l|}{ Tipo de parto } \\
\hline Normal & 69,6 & 30,4 & 0,563 & 1,32 \\
\hline Cesárea & 71,5 & 28,5 & & 1,24 \\
\hline Fórceps & 76,9 & 23,1 & & 1,00 \\
\hline \multicolumn{5}{|l|}{ Peso ao nascer } \\
\hline Baixo peso & 69,6 & 30,4 & 0,386 & 1,12 \\
\hline Peso adequado & 72,9 & 27,1 & & 1,00 \\
\hline \multicolumn{5}{|l|}{ Gestação múltipla } \\
\hline Sim & 37,5 & 62,5 & 0,043 & 2,26 \\
\hline Não & 72,4 & 27,6 & & 1,00 \\
\hline \multicolumn{5}{|l|}{ Idade gestacional } \\
\hline $\mathrm{RN}$ pré-termo & 71,0 & 29,0 & 0,876 & 1,02 \\
\hline $\mathrm{RN}$ a termo & 71,7 & 28,3 & & 1,00 \\
\hline
\end{tabular}


Tabela 3. Fatores assistenciais associados à anemia em mulheres atendidas em um Banco de Leite HumanoBelo Horizonte/MG, Brasil, 2009. Regressão de Poisson.

\begin{tabular}{lcc}
\hline \multicolumn{1}{c}{ Categorias } & RP & IC95\% \\
\hline $\begin{array}{l}\text { Mulheres que não realizaram o } \\
\text { pré-natal }\end{array}$ & 3,84 & $3,26-4,54$ \\
$\begin{array}{l}\text { Mulheres que realizaram até 3 } \\
\text { consultas pré-natais }\end{array}$ & 1,92 & $1,21-3,06$ \\
$\begin{array}{l}\text { Mulheres que tiveram gestação } \\
\text { múltipla }\end{array}$ & 2,29 & $1,25-4,19$ \\
\hline
\end{tabular}

IC: intervalo de confiança; RP: Razão de prevalência. Nota: No modelo multivariado foram contempladas as variáveis: Idade, Profissão, Estado Civil, Número de Consultas Pré-natal e Gestação Múltipla.

baixa adesão à suplementação tem se apresentado como uma grande barreira para o sucesso desta proposta ${ }^{18,19}$.

Um dos eventos relacionados à baixa adesão à suplementação do sulfato ferroso são os efeitos colaterais ocasionados, como por exemplo, náuseas, vômitos, diarreia, constipação e dor abdominal ${ }^{21}$. Além disso, um estudo comparou a dosagem de ferritina sérica em gestantes que recebiam 40, 60 e $80 \mathrm{mg}$ de ferro por dia (doses recomendadas pela OMS) e não houve diferença significativa entre as três dosagen ${ }^{22,23}$. Portanto adotar a suplementação de 40mg conforme o MS preconiza, pode ser considerada uma estratégia para diminuir os efeitos negativos da suplementação do sulfato ferroso ${ }^{22}$.

Em um estudo realizado por Sinisterra-Rodriguez $^{24}$ detectou-se a anemia em 691 grávidas atendidas no terceiro trimestre da gestação em um hospital maternidade, em São Paulo. Foram identificadas $29,2 \%$ de gestantes anêmicas. Não se observou associação entre a frequência da anemia e o atendimento pré-natal nem entre anemia e desnutrição materna, porém houve associação entre o baixo peso ao nascer e a anemia. Além disso, a hemoglobina de mulheres que usaram suplemento de ferro foi estatisticamente maior em relação àquelas que não utilizaram² ${ }^{24}$.

Em estudo realizado em 13 municípios das diferentes regiões do Brasil, a partir da adoção das medidas mencionadas, a incidência de anemia entre gestantes diminuiu de 25\% para 20\% em toda a amostra ${ }^{7}$. Entretanto, apesar do aumento no valor da hemoglobina, de $11,8 \mathrm{~g} / \mathrm{dL}$ antes da fortificação para $11,9 \mathrm{~g} / \mathrm{dL}$ após a fortificação, a prevalência de anemia continuou alta, principalmente nas regiões Norte (25\%) e Nor- deste (29\%), demonstrando baixa efetividade das medidas mencionadas ${ }^{7}$.

Outro estudo realizado em Teresina (Piauí) avaliou a concentração de hemoglobina de gestantes que deram a luz quando a fortificação ainda não era obrigatória e novamente após a implementação dessa estratégia ${ }^{23}$. O estudo encontrou que os níveis médios de hemoglobina subiram de $11,7 \mathrm{~g} / \mathrm{dL}$, antes da fortificação, para $12,4 \mathrm{~g} / \mathrm{dL}$, após a fortificação a prevalência de anemia caiu de $27,2 \%$ no grupo não fortificado para $11,5 \%$ no grupo fortificado $(p<0,001)$. Ou seja, a prevalência deste agravo gestacional antes da fortificação representava um problema de importância epidemiológica moderada e, após a fortificação, o problema passou para a categoria leve, segundo a classificação da $\mathrm{OMS}^{21,23}$.

Ademais, foi evidenciado, no estudo atual, associação entre a presença de anemia e fatores assistenciais, com destaque para a ausência ou baixo número de consultas pré-natais ${ }^{25,26}$. Entre as mulheres da pesquisa, 53,6\% apresentaram resultado muito distante da preconização do MS que, através do Programa de Humanização do Pré-Natal e Nascimento ${ }^{27}$, implantado no ano 2000, orienta pelo menos seis consultas pré-natais para toda gestação a termo, com início do acompanhamento ainda no primeiro trimestre da gravidez ${ }^{25,26}$. $\mathrm{O}$ maior número de consultas possibilita a avaliação das intercorrências clínico-obstétricas mais frequentes ${ }^{26}$. Além disso, o início precoce do acompanhamento à gestante associa-se a benefícios tanto para a mãe quanto para o bebê, diminuindo a incidência de baixo peso, peso insuficiente ao nascer e prematuridade, bem como favorecendo a prática de aleitamento materno (AM) $)^{22,26,27}$.

A literatura aponta que mulheres grávidas que recebem assistência no início da gestação e comparecem a mais consultas de pré-natal tendem a apresentar melhores resultados maternos e perinatais comparado às gestantes que o iniciam tardiamente ou entre aquelas que tiveram as consultas mais concentradas em um único trimestre ${ }^{28,29}$.

Ressalta-se, que a consulta pré-natal propicia o acompanhamento dos parâmetros bioquímicos da gestante, com o objetivo de identificar carências nutricionais precocemente e estabelecer as intervenções cabíveis ${ }^{29}$. Adicionalmente, é importante que a orientação nutricional seja realizada durante todo o pré-natal, ou até mesmo no período anterior à gestaçã $0^{6,30}$. Este acompanhamento tem um papel fundamental no resultado obstétrico, além de ser um importante aliado no combate à alimentação inadequada, pois incentiva a adoção de hábitos alimentares saudáveis com o intuito de 
melhorar o consumo de nutrientes essenciais à gestação, como o ferro, atuando de forma preventiva e terapêutica contra a anemia ${ }^{2,31}$.

No presente estudo, essa carência nutricional também foi mais prevalente entre as mulheres em gestação múltipla, provavelmente devido à demanda aumentada de ferro, em razão de uma hematopoiese elevada, causada pela rápida expansão dos tecidos que ocorre neste período e, de maneira mais acentuada, em gestações múltiplas para atender as necessidades dos fetos ${ }^{31}$. Além disso, o estudo de Assunção et al. $^{32}$ também relacionou a gestação ao maior risco de prematuridade, demonstrando que esse evento requer maior atenção e cuidado por parte dos serviços de saúde ${ }^{32}$.

No atual trabalho, a gestação múltipla foi observada em $0,9 \%$ de todas as gestantes, sendo que destas, 62,5\% apresentaram hemoglobina abaixo dos valores de referência. Já nos achados de Graner e Barros ${ }^{33}$ que, a partir dos estudos conduzidos em 131 prontuários de gestantes em um hospital e maternidade de São Paulo, detectou-se que $29,1 \%$ da amostra apresentavam gestação múltipla, sendo que $12,2 \%$ possuíam anemia ${ }^{33}$. Tal achado coloca as gestações gemelares como um grupo mais susceptível à anemia ferropriva e demais carências nutricionais devido, principalmente, às especificidades deste tipo de gravidez, entretanto, os achados atuais ainda são inconsistentes no que concerne essa questão $0^{33,34}$.

Estudos descritos na literatura concluíram que cuidados pré-natais intensivos para as mulheres com gravidez múltipla foram efetivos na promoção de desfechos gestacionais positivos e prevenção de agravos à saúde do binômio mãefilho, o que reforça a importância da realização do pré-natal individualizado e adequado ${ }^{34,35}$.

Ressalta-se a limitação de não se apontar as relações de causalidade entre as variáveis no presente estudo, uma vez que pesquisas de delinea- mento transversal não favorecem a identificação da ordem de exposição/resultado. Além disso, os dados disponíveis não possibilitaram classificar o tipo de anemia, porém, outros trabalhos apontam que a anemia ferropriva é a mais prevalente entre gestantes e nutrizes ${ }^{10}$. Ademais não foi avaliado o uso do sulfato ferroso e a ingestão alimentar de ferro entre as mulheres devido à ausência dessa informação no material investigado ${ }^{5}$.

Cumpre destacar, no entanto, que apesar das limitações descritas, os resultados deste estudo permitiram identificar fatores assistenciais e gestacionais associados à anemia em nutrizes, informações fundamentais para delinear intervenções direcionadas à saúde dessa população. Adicionalmente, destaca-se que o estudo foi conduzido dentro de um banco de leite humano, em uma maternidade pública, sendo este, um cenário real do serviço de saúde.

Por fim, o perfil das gestantes brasileiras associado aos indicadores de saúde materna e ao perfil das nutrizes estudadas é semelhante, considerando as desigualdades sociais, refletindo as condições diferenciadas de vida e de acesso a recursos sociais entre diversos grupos da população e a realidade da saúde materno-infantil no país ${ }^{35}$.

\section{Conclusão}

Os resultados apontaram maior prevalência de anemia entre as mulheres que não realizaram o pré-natal de modo apropriado e entre aquelas que tiveram gestações múltiplas. Assim, destaca-se a importância do pré-natal como fator de prevenção à anemia e a necessidade de ampliação das intervenções ora propostas pelo governo para esta temática a fim de melhorar a qualidade da atenção à saúde da gestante e da nutriz.

\section{Colaboradores}

LB Ferreira: Redação, desenvolvimento e análise estatística. TM Sousa, LF Melo e MEF Melo: Redação e desenvolvimento. C Miranda: Análise estatística. SCL Pereira: Coleta de dados e revisão. LC Santos: Revisão e aprovação final do manuscrito. KAM Notaro: revisão do manuscrito. 


\section{Referências}

1. World Health Organization (WHO). The global prevalence of anemia in 2011. Geneva: WHO; 2015. [acessado 2015 Maio 9]. Disponível em: http://apps.who.int/iris/ bitstream/10665/177094/1/9789241564960_eng.pdf

2. Pessoa LDS, Saunders C, Belfort GP, Silva LBGD, Veras LS, Esteves APVDS. Evolução temporal da prevalência de anemia em adolescentes grávidas de uma maternidade pública do Rio de Janeiro. Rev bras ginecol obstet 2015; 37(5):208-215.

3. Camargo RMSD, Pereira RA, Yokoo EM, Schirmer J. Factors associated with iron deficiency in pregnant women seen at a public prenatal care service. Rev Nutr 2013; 26(4):455-464.

4. Aragão FKS, Almeida ALD, Nunes SFL. Prevalência e fatores associados à anemia em gestantes atendidas em uma maternidade pública no município de Imperatriz, Maranhão. J Management Primary Health Care 2014; 4(3):183-190.

5. Brandão AHF, Cabral MA, Cabral ACV. A Suplementação de ferro na gravidez: orientações atuais. Rev Femina 2011; 39(5):285-289.

6. Rodrigues LP, Jorge SRPF. Deficiência de ferro na gestação, parto e puerpério. Rev Bras Hematol Hemoter 2010; 32(2):53-56.

7. Fujimori E, Sato APS, Szarfarc SC. Anemia em gestantes brasileiras antes e após a fortificação das farinhas com ferro. Rev Saude Publica 2011; 45(6):1027-1035.

8. Morse ML, Fonseca SC, Gottgtroy Cl, Waldmann CS, Gueller E. Morbidade materna grave e near misses em hospital de referência regional. Rev Bras Epidemiol 2011; 14(2):310-322.

9. Vettore MV, Dias M, Leal MC. Avaliação da qualidade da atenção pré-natal dentre gestantes com e sem história de prematuridade no Sistema Único de Saúde no Rio de Janeiro, Brasil. Rev Bras Saúde Matern Infan 2013; 13(2):89-100.

10. Oliveira ACM, Barros AMR, Ferreira RC. Fatores de risco associados à anemia em gestantes da rede pública de saúde de uma capital do Nordeste do Brasil. Rev Bras Ginecol Obstet 2015; 37(11):505-511.

11. Brasil. Ministério da Saúde (MS). Secretaria de Vigilância em Saúde. Secretaria de Atenção à Saúde. Manual normativo para profissionais de saúde de maternidades - referência para mulheres que não podem amamentar. Brasília: MS; 2005. [acessado 2016 Maio 9]. Disponível em: http://www.ibfan.org.br/documentos/outras/MSmanualHIVeAM2005.pdf

12. Ribeiro ER, Guimarães AMD, Bettiol H, Lima DD, Almeida MLD, Souza L, Gurgel RQ. Risk factors for inadequate prenatal care use in the metropolitan area of Aracaju, Northeast Brazil. BMC pregnancy and childbirth 2009; 9(1):31-38.

13. Spong CY. Defining "term" pregnancy: Comitee Opinion. The American College of Obstetricians and Gynecologists Committee on Obstetric Practice Society for Maternal-Fetal Medicine 2013; 579:2445-2446.
14. World Health Organization (WHO). Young People's Health - a Challenge for Society. Report of a WHO Study Group on Young People and Health for All.Technical Report Series 731. Geneva: WHO; 1986. [acessado 2016 Maio 9]. Disponível em: http://apps.who.int/iris/bitstream/10665/41720/1/WHO_TRS_731.pdf

15. Accioly E, Saundes C, Lacerda EMA. Nutrição em obstetrícia e pediatria. Rio de Janeiro: Cultura Médica Guanabara, 2009.

16. Lima LC, Reis NT. Interpretação de exames laboratoriais aplicados à Nutrição Clínica. Rio de Janeiro: Editora Rubio; 2012.

17. World Health Organization (WHO). Iron deficiency anemia: assessment, prevention and control - a guide for program managers. Geneva: WHO; 2001. [acessado 2015 Out 20]. Disponível em: http://apps.who.int/iris/ bitstream/10665/66914/1/WHO_NHD_01.3.pdf?ua=1

18. Brasil. Ministério da Saúde (MS). Programa Nacional de Suplementação de Ferro: manual de condutas gerais. Brasília: MS; 2013. [acessado 2016 Maio 9]. Disponível em: http://bvsms.saude.gov.br/bvs/publicacoes/manual_suplementacao_ferro_condutas_gegera.pdf

19. Bhutta ZA, Das JK, Rizvi A, Gaffey MF, Walker N, Horton S. Maternal and Child Nutrition Study Group. Evidence-based interventions for improvement of maternal and child nutrition: what can be done and at what cost? Lancet 2013; 382(9890):452-477.

20. Brasil. Ministério da Saúde (MS). Pesquisa nacional de demografia e saúde da criança e da mulher - PNDS 2006: dimensões do processo reprodutivo e da saúde da criança. Brasília: MS; 2009.

21. Organização Mundial da Saúde (OMS). Diretriz: Suplementação diária de ferro e ácido fólico em gestantes. Genebra: OMS; 2013. [acessado 2016 Maio 9]. Disponível em: http://189.28.128.100/dab/docs/portaldab/documentos/guia_gestantes.pdf

22. Brasil. Ministério da Saúde (MS). Caderno de Atenção Básica no 32. Atenção ao pré-natal de baixo risco. Brasília: MS; 2012. [acessado 2016 Maio 9]. Disponível em: http://www.scielosp.org/pdf/csc/v20n6/14138123-csc-20-06-1815.pdf

23. Filho MDS, Damascena CVX, Szarfac SC, Fujimori E, Mota AAA, Reis RS. Fortificação das farinhas com ferro e controle da anemia em gestantes de Teresina, Piauí, Brasil. Rev Nutr 2011; 24(5):679-688.

24. Sinisterra-Rodriguez OT. Anemia nutricional en el embarazo. Rev Hosp Niño (Panamá) 1990; 9(2):121-124.

25. Buzzo ML, Carvalho MFH, Tiglea P, Arauz LJ, Kumagai EE, Arakaki RM. Monitoramento de farinha de trigo e de milho fortificadas com ferro. Rev. Instituto Adolfo Lutz 2012; 71(4):645-649.

26. Silva EP, Lima RT, Costa MJC, Batista Filho M. Desenvolvimento e aplicação de um novo índice para avaliação do pré-natal, Rev Panam Salud Publica 2013; 33(5):356-362. 
27. Andreucci CB, Cecatti JG. Desempenho de indicadores de processo do Programa de Humanização do Pré-natal e Nascimento no Brasil: uma revisão sistemática. Cad Saude Publica 2011; 27(6):1053-1064.

28. Duarte HSJ. Motivos que levam as gestantes a fazerem o pré-natal: Um estudo das representações sociais. Rev Ciência Enferm 2012; 18(2):75-82.

29. Corrêa CRH, Bonadio IC, Tsunechiro MA. Avaliação normativa do pré-natal em uma maternidade filantrópica de São Paulo. Rev Escol Enferm USP 2011; 45(6):1293-1300.

30. Domingues RMSM, Araújo HZM, Dias MAB, Carmo Leal M. Avaliação da adequação da assistência pré-natal na rede SUS do Município do Rio de Janeiro, Brasil. Cad Saude Publica 2012; 28(3):425-437.

31. Santos MM, Cavalcante DB, Lima JN, Baião MR, Saunders C. Impact of an intervention nutrition program during prenatal on the weight of new borns from teenage mothers. Nutrición Hospitalaria 2013; 28(6):19431950.

32. Assunção PL, Novaes HMD, Alencar GP, Oliveira ASM, Almeida MF. Fatores associados ao nascimento pré-termo em Campina Grande, Paraíba, Brasil: um estudo caso-controle. Cad Saude Publica 2012; 28(6):10781090.

33. Graner VR, Barros SMO. Complicações maternas e ocorrências neonatais associadas às gestações múltiplas resultantes de técnicas de reprodução assistida. Rev Escol Enferm USP 2009; 43(1):103-109.

34. Dodd JM, Crowther CA. Specialised antenatal clinics for women with a multiple pregnancy for improving maternal and infant outcomes. Cochrane Database Syst Rev 2012; (8):CD005300.

35. Xavier RB, Jannotti CB, Silva KS, Martins AC. Risco reprodutivo e renda familiar: análise do perfil de gestantes. Cien Saude Colet 2013; 18(4):1161-1171.

Artigo apresentado em 22/06/2016

Aprovado em 02/03/2017

Versão final apresentada em 04/03/2017 
